\title{
Toward Reducing COPD Hospitalization
}

COPD is a chronic inflammatory process in the airways (and often in the adjacent alveolar spaces) that afflicts millions of patients in the United States alone. Pathologic changes are generally irreversible, and the disease is characterized by progressive respiratory deterioration punctuated by exacerbations. These exacerbations can be triggered by any of a large number of factors, although the most common triggers are infections. Patients present with signs and symptoms ranging from simple increases in cough, sputum, or dyspnea to full-blown respiratory failure necessitating hospitalization and respiratory support.

COPD exacerbations are associated with an accelerated decline in respiratory function, and exacerbation frequency or intensity is a powerful predictor of future episodes and ultimate mortality. Indeed, exacerbation frequency is the strongest single factor related to future exacerbations, and mortality rates increase 3 -fold as exacerbation frequency increases from 0 to 3 or more per year. ${ }^{1}$ Exacerbations result in $>700,000$ hospitalizations per year in the United States alone. Importantly, roughly 1 in 5 COPD hospitalizations is followed by a readmission within $30 \mathrm{~d}$, resulting in cost estimates of more than $\$ 15$ billion per year. ${ }^{2}$

Because of these health consequences and costs, it should not be surprising that in the last decade the medical community (along with payers) have launched numerous initiatives to reduce exacerbations, with a particular focus on reducing readmissions due to exacerbation. These initiatives involve improving medication regimens and adherence, smoking cessation, pulmonary rehabilitation, close follow-up by clinicians following discharge, assessments for oxygen supplementation and noninvasive ventilation, and psychosocial support structures. ${ }^{3}$ There is increased focus on patient involvement and self-management, which can be augmented with in-patient COPD "navigators"4 and out-patient telehealth programs. ${ }^{5}$ Payers, led by the U.S. Center for Medicare and Medicaid Services (CMS) have supported these efforts in addition to imposing a $3 \%$ pen-

The author has disclosed no conflicts of interest.

Correspondence: Neil R MacIntyre MD FAARC, Division of Pulmonary and Critical Care Medicine, Duke University Medical Center, Box 3911, Durham, NC 27710. E-mail: neil.macintyre@duke.edu.

DOI: $10.4187 /$ respcare.07491 alty on all Medicare payments to hospitals who have excessive COPD readmission rates. ${ }^{5,6}$

\section{See the Original Study on Page 1}

Efforts to reduce COPD exacerbation readmissions have had only limited success. ${ }^{2}$ Part of the reason is the complexity of COPD management, that is, assuring that the components COPD management are indeed occurring. Another factor limiting success can be difficulty obtaining patient and family buy-in. COPD disease management is a partnership, and patient engagement is critical. Finally, COPD readmissions often involve the numerous comorbidities seen in patients with COPD. In one study, the principal diagnosis associated with the 30-d readmission of a COPD patient was related to COPD (ie, "COPD and bronchiectasis," "respiratory failure" or "pneumonia") less than half the time. ${ }^{2}$ Better COPD management may have little effect on these non-COPD issues; indeed, the link between reduced readmissions in patients with COPD and better overall outcomes is not well established. $^{5}$

Non-patient-related factors can also hamper the success of efforts to reduce COPD readmission rates. For example, payer efforts to reward readmission reductions may backfire if a needed hospitalization is blocked and a worsening episode of respiratory failure develops. This may affect underserved populations disproportionately. Payer policies can also produce perverse incentives-eg, an admission may generate more hospital income than the reward for preventing it. These policies might also foster deliberate miscoding efforts and encourage hospital policies to transfer patients to other facilities. ${ }^{5}$

In this issue of Respiratory CARE, Zafar et $\mathrm{al}^{7}$ address a unique, understudied population of patients experiencing an exacerbation of COPD: patients admitted to an emergency department observation unit. These are patients with clear acute respiratory compromise but for whom a $24-36-h$ period of therapy and close monitoring is deemed appropriate before a decision is made to hospitalize or discharge. The research question builds on the studies noted above, which generally support the concept that carefully designed and executed care bundles can potentially reduce $30-\mathrm{d}$ rehospitalization rates. This study goes a step further: Can a well-designed, short-course "bundle" reduce emergency department re- 
visits in the next $30 \mathrm{~d}$ ? The question is an important one as the authors note a $48 \%$ all-cause emergency department revisit rate in their baseline population.

The results are important. First, the authors note that a bundle designed for $<36 \mathrm{~h}$ of application can be implemented successfully $>90 \%$ of the time. Second, after bundle initiation, the 30-d all-cause emergency department revisit rate was noted to drop significantly to $30 \%$. Third, the bundle succeeded even without components that others have shown to be important such as pulmonary rehab, $\mathrm{O}_{2}$ assessment, psychosocial assessment, and home noninvasive ventilation assessment. This highlights the importance of some of the key components of this bundle, such as medication management and scheduled follow-up.

There are limitations to this study. First, it is a single-center study utilizing only a pre- and postimplementation study design. There was no concurrent control group. Second, by necessity, a short-course bundle does not allow for inclusion of other interventions that take longer to implement. Finally, data are reported only on subjects admitted to an emergency department observation unit. Patients with direct admission (ie, bypassing the emergency department observation unit) and patient mortality (both hospitalized or at home) are not reported.

Despite these limitations, this study adds further support to the concept that the best management strategies for COPD are multidisciplinary and patient-focused, and they must involve multiple team members. Moreover, this work reinforces the notion that COPD care must be integrated across the spectrum from chronic home management to out-patient clinics to acute care facilities.
Neil R MacIntyre

Division of Pulmonary and Critical Care Medicine Duke University Medical Center

Durham, North Carolina

\section{REFERENCES}

1. Soler-Cataluña JJ, Martínez-García MA, Román Sánchez P, Salcedo E, Navarro M, Ochando R. Severe acute exacerbations and mortality in patients with chronic obstructive pulmonary disease. Thorax 2005; 60(11):925-931.

2. Jacobs DM, Noyes K, Zhao J, Gibson W, Murphy TF, Sethi S, OchsBalcom HM. Early hospital readmissions after an acute exacerbation of COPD in the Nationwide Readmissions Database. Ann Am Thorac Soc 2018;15(7):837-845.

3. Prieto-Centurion V, Markos MA, Ramey NI, Gussin HA, Nyenhuis $\mathrm{SM}$, Joo MJ, et al. Interventions to reduce re-hospitalizations after COPD exacerbations: a systematic review. Ann Amer Thorac Soc 2014;11(3):417-424.

4. Euceda G, Kong WT, Kapoor A, Hokanson JE, Dilauro P, Ogunnaike $\mathrm{R}$, Chronakos J. The effects of a comprehensive care management program on readmission rates after acute exacerbation of COPD at a community-based academic hospital. Chronic Obstr Pulm Dis 2018; 5(3):185-192.

5. Shah T, Press VG, Huisingh-Scheetz M, White SR. COPD readmissions: addressing COPD in the era of value-based health care. Chest 2016;150(4):916-926.

6. Feemster LC, Au DH. Penalizing hospitals for chronic obstructive pulmonary disease readmissions. Am J Respir Crit Care Med 2014; 189(6):634-639.

7. Zafar MA, Loftus TM, Palmer JP, Phillips M, Ko J, Ward S, et al. COPD care bundle in emergency department observation unit reduces emergency department revisits. Resp Care 2020;65(1):1-10. 